\title{
Super-Resolution Fluorescence in Electron Micrographs Using In-Situ Integrated Microscopy
}

Jacob P. Hoogenboom

Imaging Physics, Delft University of Technology, Delft, the Netherlands.

Superresolution (SR) fluorescence has revolutionized the field of optical microscopy by achieving molecular localization well below the diffraction limit of light [1]. However, the non-labelled structural context of the cell remains dark, while this context may be crucial in understanding how biological molecules function. Correlation of SR data with structural images obtained with electron microscopy (EM) has been demonstrated [2], but requirements for SR microscopy are often in conflict with those for EM. Moreover, registration of separate SR and EM images may be difficult and introduce additional errors on top of the optical localization accuracy. I will present alternative approaches to achieve molecular localization within the cellular context using integrated light and electron microscopy. In our system, a high numerical aperture fluorescence microscope is integrated inside a scanning EM in such a way that the electron beam can be positioned anywhere within the field of view of the fluorescence microscope [3], as indicated in Figure 1. This allows to record fluorescence-guided EM snapshots during live-cell dynamics [4], as well as to achieve SR within a high-resolution EM image by monitoring electron-induced modifications of the fluorescence signal from labelled bio-molecules.

Live cells are kept in the integrated microscope using a dedicated holder that allows to keep samples in liquid in the vacuum chamber. A $40 \mathrm{~nm}$ silicon nitride window, like typically used in in-situ (S)TEM, allows recording of back-scattered electron signal in the SEM (Figure 2a). The dynamics of live cellular processes can then be followed with fluorescence microscopy, in our case using bio-conjugated quantum dots. Based on the live-cell fluorescence observations, a region of interest for SEM is selected. We thus only expose our region of interest to the electron beam and moreover delay this exposure until live dynamics has been observed. A single SEM snapshot suffices to embed the fluorescence observations with structural context (see Figure 2b). Moreover, these high-resolution snapshots reveal the quantum dots during uptake at resolution far below that of the fluorescence microscope and at high spatial densities.

References:

[1] B Huang, M Bates and X Zhuang, Annual review of biochemistry 78 (2009), p. 993.

[2] P de Boer, JP Hoogenboom and BNG Giepmans, Nature Methods 12 (2015), p. 503.

[3] AC Zonnevylle et al, Journal of Microscopy 252 (2013), p. 58.

[4] N Liv et al, ACS Nano 10 (2016), p. 265. 


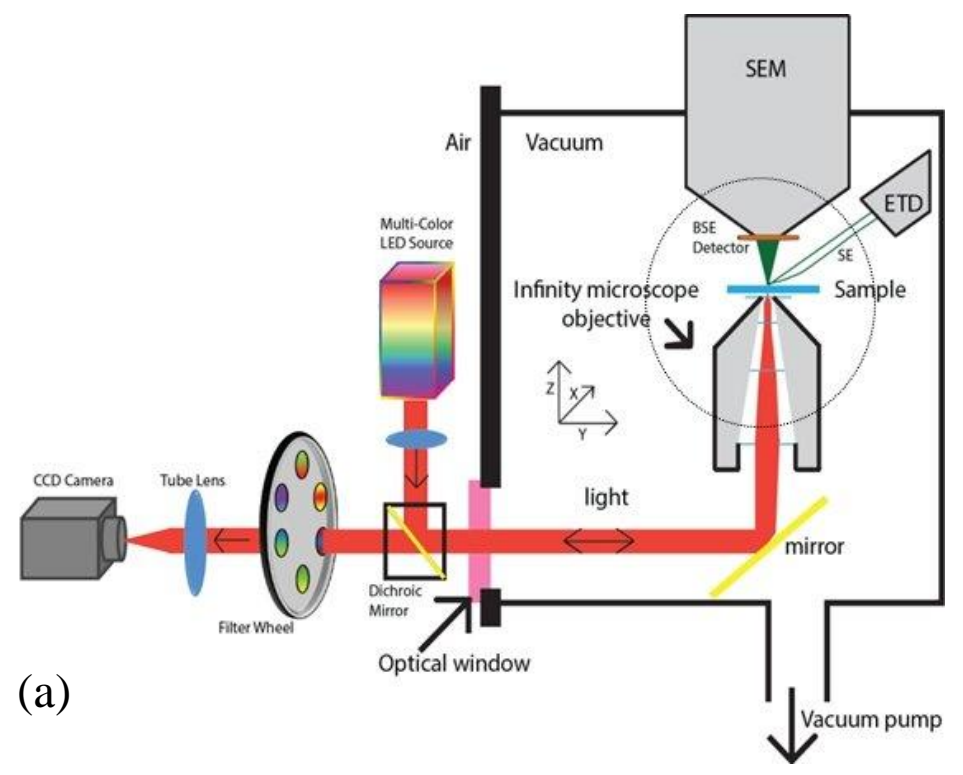

(b)

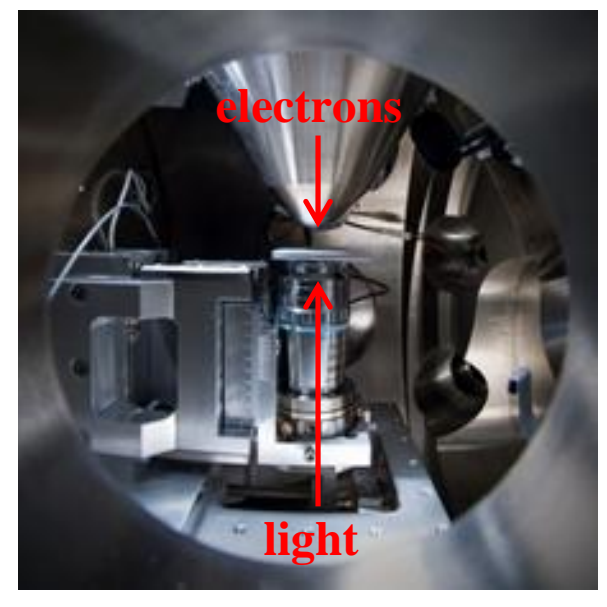

Figure 1. (a) Schematic layout of the integrated fluorescence - scanning electron microscope. Objective lens and mirror for fluorescence microscopy are positioned inside the vacuum chamber, underneath the sample. All other fluorescence microscope components are outside the vacuum with light guided through a glass window. (b) Inside view of the microscope (circled area in (a)), where sample stage, and light and electron objective lenses are visible.

(a)

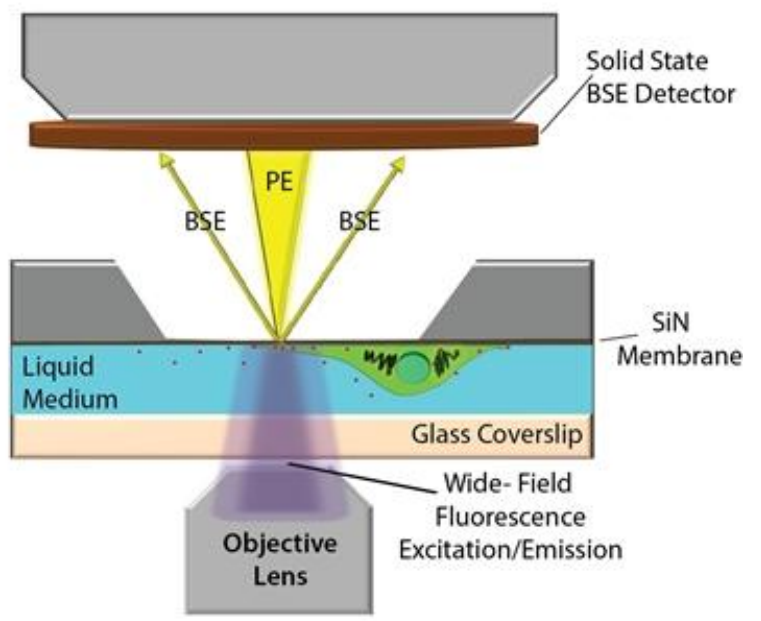

(b)

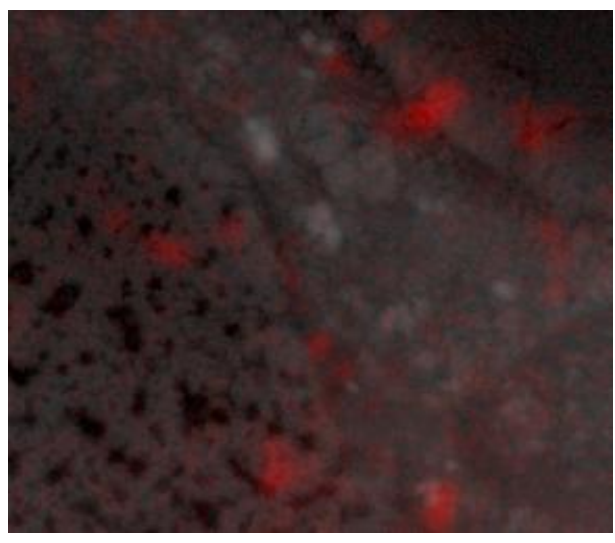

Figure 2 (a) Schematic indication of the set-up for live-cell fluorescence microscopy. Cells are cultured on a $40 \mathrm{~nm}$ silicon nitride window, which is placed in a encapsulation to keep the cell in liquid environment in the integrated microscope. (b) Example of fluorescence from bio-conjugated quantum dots (in red) overlaid with a scanning electron microscopy snapshot recorded during quantum dot uptake. 